\title{
Kernos
}

Revue internationale et pluridisciplinaire de religion grecque antique

18 | 2005

Varia

\section{Influenze egizie nella Descriptio orbis di Dionisio d'Alessandria?}

\section{Eugenio Amato}

\section{(2) OpenEdition \\ Journals}

\section{Edizione digitale}

URL: http://journals.openedition.org/kernos/909

DOI: 10.4000/kernos.909

ISSN: 2034-7871

\section{Editore}

Centre international d'étude de la religion grecque antique

\section{Edizione cartacea}

Data di pubblicazione: 1 gennaio 2005

Paginazione: 97-111

ISSN: 0776-3824

Notizia bibliografica digitale

Eugenio Amato, «Influenze egizie nella Descriptio orbis di Dionisio d'Alessandria? », Kernos [En ligne] 18 | 2005, mis en ligne le 08 juillet 2011, consulté le 05 mai 2019. URL : http:// journals.openedition.org/kernos/909; DOI : 10.4000/kernos.909 


\title{
Influenze egizie nella Descriptio orbis di Dionisio d'Alessandria?
}

\footnotetext{
Résumé: Des influences égyptiennes dans la Descriptio orbis de Denys d'Alexandrie? L'examen attentif des composantes laudatives et du contenu du proème de la Descriptio orbis de Denys le Périégète prouve que le poète originaire d'Alexandrie a été influencé dans son ouvre même par la religion égyptienne. En particulier, derrière l'éloge de l'Océan parât se cacher un hymne à la louange du Nun primordial.
}

\begin{abstract}
Egyptian Influences in the Descriptio orbis by Dionysius of Alexandria? The thorough analysis of the laudatory components and contents of the proem by Dionysius Periegetes' Descriptio orbis shows that the Alexandrian poet was also influenced in his own poem by the Egyptian religion. In particular, behind the encomium of the Ocean, it looks as if the poet is hiding a hymn to the primeval Nun.
\end{abstract}

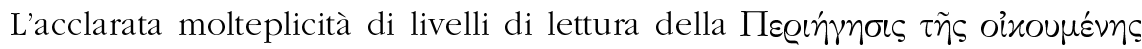
di Dionisio d'Alessandria, poema innodico-didattico di argomento geografico in cui si riversano le marche letterarie tipiche della letteratura greca imperiale

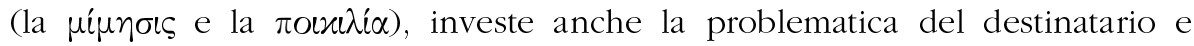
dell'ambiente culturale al quale il poeta di età adrianea s'indirizza; problematica, invero, tanto più urgente e rilevante quanto maggiore è l'aderenza del poeta allo statuto della poesia didascalica in cui tale testo è iscritto ${ }^{1}$. Come afferma Servio nel proemio al suo commento alle Georgiche di Virgilio: Et hi libri didascalici sunt, unde necesse est ut ad aliquem scribantur; nam praeceptum et doctoris et discipuli personam requirit. Il rapporto tra un doctor e un discipulus è una costante strutturale del genere didascalico, un genere centrale nell'esperienza della cultura classica.

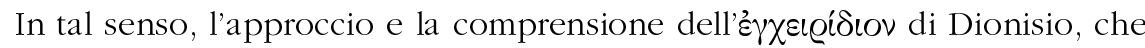
vuole senz'altro essere nelle immediate intenzioni del suo autore la descrizione di un $\pi i v \alpha \xi$ virtuale, non può non tener conto di alcune istanze meno evidenti, ma altrettanto significative nel processo di comunicazione che l'autore instaura col suo lettore, soprattutto laddove si consideri l'origine alessandrina di Dionisio.

La domanda cui vorrei tentare di rispondere è la seguente: qual era il background culturale di Dionisio? Vi sono elementi all'interno della Descriptio

\footnotetext{
1 Su tale aspetto, vedi C. JACOB, "Le sujet et le texte. Sur l'identité de Denys le Périégète", Lalies 4 (1984), p. 215-239: 235; id., La Description de la Terre habitée de Denys d'Alexandrie ou la leçon de géographie, Paris, 1990, praes. p. 11-41; F. COCCARO ANDREOU, "I molteplici livelli di lettura della Periegesi della Terra abitata di Dionisio d'Alessandria", in E. AmaTO, F. D'Avino, A. Esposito (ed.), Primum Legere. Annuario della Delegazione della Valle del Sarno dell'A.I.C.C., 1, Salerno, Helios, 2002, p. 105-133.
} 
orbis che permettono di individuare i legami del poeta con la sua patria d'origine? Fermo restando lo statuto africano del Periegeta, è possibile, cioè, scorgere nel suo poema tratti tipici della cultura egizia, che riflettano anche una destinazione alessandrina?

La questione non è certo peregrina, soprattutto se si considera il caso omologo di altri autori antichi, quali Teocrito, Callimaco ed Apollonio Rodio, fontes privilegiati di Dionisio e per i quali si è dimostrato in parte un rapporto fra ideologia dinastica e poesia ${ }^{2}$. Né sarebbe l'unico caso di influenze orientali nell'epos didascalico classico, attestato anche in poemi filosofici di rilevanza particolare, in cui l'autore si sforza di offrire al destinatario l'esperienza di un viaggio non comune, che lo rende unico da ogni altro tentativo simile ${ }^{3}$.

Sfortunatamente, nel caso di Dionisio, al di là della generica informazione della sua origine alessandrina che si legge nell'acrostico dei v. 109-134 (EMH $\triangle$ IONY $\Sigma[\text { I]IOY TSN ENTO } \Sigma \text { (APOY })^{4}$, non abbiamo alcuna notizia circa il suo ruolo nell'Alessandria d'Egitto di età adrianea'. Né tanto meno gli 'appelli'

\footnotetext{
${ }^{2}$ In particolare per Teocrito si ricordi l'importante saggio di F.T. GRIFFITHS, Theocritus at Court, Leiden, 1979; $c f$., inoltre, J. Duchemin, "Quelques analogies égyptiennes dans les poèmes de Théocrite", IL 1 (1954), p. 25-31 (= Mythes grecs et sources orientales, Paris, 1995, p. 13-25); O. FAYEZ RIAD, "Théocrite entre la Sicile et l'Égypte", in Roma e l'Egitto nell'antichità classica. Cairo, 6-9 Febbraio 1989. Atti del I Congresso Internazionale Italo-Egiziano, Roma, 1992, p. 305315: 309-314. Per Callimaco, vedi di recente S. STEPHENS, "Callimachus at Court", in M.A. HARDER, R.F. Regtuit, G.C. WAKKer (ed.), Genre in Hellenistic Poetry, Groningen, 1998, p. 167-185, ma anche G. CAPOvilla, Callimaco, I, Roma, 1967, p. 84-143 e L. LeHnus, "Callimaco tra la polis e il regno", in G. CAMbiano, L. CANFORA, D. LANZA, Lo spazio letterario di Grecia antica, I/2, Roma, 1993, p. 75-105. Quanto ad Apollonio Rodio, vedi R.L. HunTER, The Argonautica of Apollonius. Literary Studies, Cambridge, 1993, p. 152-169 e O. Vox, "Noterelle di epica ellenistica", Rudiae 11 (1999), p. 163-172: 164-165.

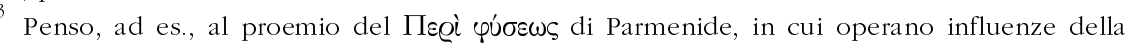
cultura mesopotamica: vedi L.D. STEELE, "Mesopotamian Elements in the Proem of Parmenides? Correspondences Between the Sun-Gods Helios and Shamash", CQ 52 (2002), p. 583-588. Per ulteriori influssi negli Inni omerici ed in Esiodo, vedi Ch. PENGLASE, Greek Myths and Mesopotamia. Parallels and Influence in the Homeric Hymns and Hesiod, London / New York, 1994.

4 L'acrostico, assieme a quello dei v. 513-532, fu scoperto da G. LEUE, "Zeit und Heimath des Periegeten Dionysios", Philologus 42 (1884), p. 175-178. Per la corretta lettura dello stesso bisogna forse considerare un'aggiunta il verso 118. Che così stiano effettivamente le cose potrebbe dimostrarlo l'assenza del verso in alcuni manoscritti antichi della Descriptio e nelle parafrasi latine di Prisciano e Avieno ( $c f$. I.On. Tsavari, Histoire du texte de la Description de la Terre de Denys le Périégète, Ioannina, p. 1990, 28, n. 19); ma la certezza non è assoluta : vedi per una discussione critica E. AMATO, "Note esegetiche e testuali alla Descriptio orbis di Dionisio d'Alessandria (III)", Göttinger Forum für Altertumswissenschaft 7 (2004), p. 1-9: 2-3 [webdoc.sub.gwdg.de/edoc/p/gfa/ 7-04/amato.pdf]).

Il padre, stando alla Suda ( $\Delta 1173=$ I, p. 109-110 ed. ADLER), fu direttore di biblioteche e capo degli uffici ab epistulis, a legationibus ed a responsis (su tali termini, vedi H.I. MASON, Greek

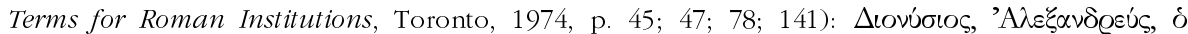

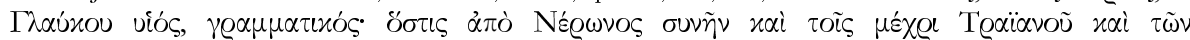

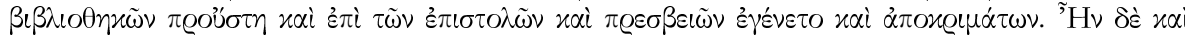

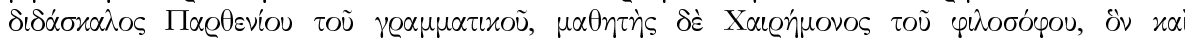
$\delta \iota \varepsilon \delta \varepsilon \xi \alpha \tau O$ év 'A $\lambda \varepsilon \xi \alpha \nu \delta \varrho \varepsilon i \alpha$. Per l'epoca in cui visse il poeta (che è quella di Adriano), recentemente contrastata da H. White, "On the Date of Dionysius Periegetes", Orpheus n.s. 22/1-2
} 
al lettore, disseminati in vario modo all'interno del suo poema, consentono di costruirci un'immagine precisa del pubblico cui il poeta s'indirizzava ${ }^{6}$.

È innegabile, come avevano già notato i commentatori antichỉ, la preferenza accordata dal Periegeta alla Libia, la cui descrizione anticipa quella degli altri due continenti emersi, ed in particolar modo dell'Egitto e della Valle del Nilo, cui sono consacrati ben 49 versi (v. 221-269) sui 1184 dell'intero poema.

Alcune delle informazioni contenute in questi potrebbero rilevarsi di estrema importanza per stabilire la cronologia del nostro poeta ${ }^{8}$. In ogni caso, essi mostrano un interesse fortemente sentito, che esprime in termini senz'altro eulogistici le virtù del paese natio, benché senza mai nominarlo?,

(2001), p. 288-290, vedi E. AMATo, "Per la cronologia di Dionisio il Periegeta", RPh 77/1 (2003), p. 7-16.

Vedi Coccaro ANDreou, l.c. (n. 1), p. 128-129. Sulla problematica, vedi in generale M. Citront, "Le raccomandazioni al lettore: apostrofe al libro e contatti con il destinatario", Maia 38 (1986), p. 111-146. In particolare, per il genere didascalico, di estremo interesse sono i saggi raccolti da A. Schiesaro, Ph. Mitsis, J. Strauss Clay (ed.), Mega nepios. Il destinatario nell'epos didascalico, Pisa, 1993 (= MD 31) ed il lucido articolo di E. Romano, "Gli appelli al lettore negli Astronomica di Manilio", Pan 6 (1978), p. 115-125.

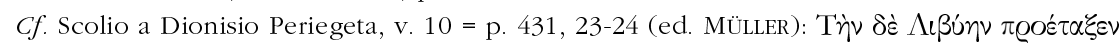

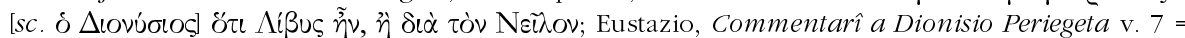

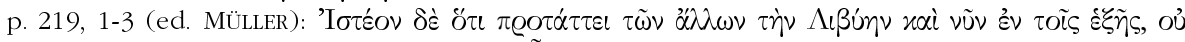

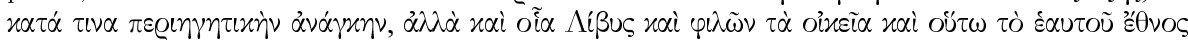
$\tau \tilde{\omega} \nu \lambda \circ \iota \pi \tilde{\omega} \nu \pi \varrho O \tau \iota \theta \varepsilon ́ \mu \varepsilon v o s$.

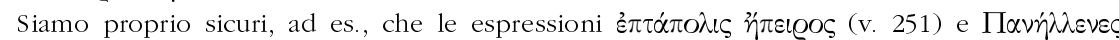
(v. 333) per indicare rispettivamente l'Egitto ed i Greci, così come l'allusione al Serapeo (v. 255)

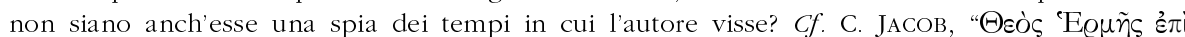

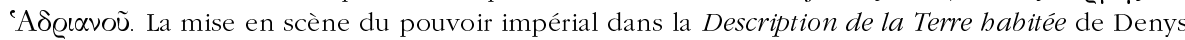
d'Alexandrie", CCG 2 (1991), p. 43-53: 44, n. 3, il quale giustamente ricorda che da Adriano venne

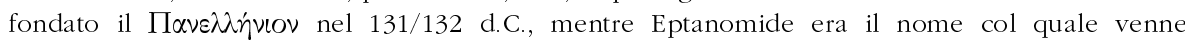
designata in epoca adrianea l'epistrategia del medio Egitto, tra la Tebaide e il Delta, e D.D. Greaves, Dionysius Periegetes and the Hellenistic Poetic and Geographical Traditions, PhD., Stanford University, 1985, p. 17-18, che sottolinea come il Serapeo, decaduto in tarda epoca ellenistica (vedi Strabone, XVII, 1, 10), rifiorisca in età imperiale, proprio grazie a Traiano ed Adriano (vedi anche U. BernaYs, Studien zu Dionysius Periegetes, diss., München, 1905, p. 20). In particolare, la connessione tra Adriano ed il Serapeo è confortata dall'evidenza dei documenti epigrafici e numismatici: cf. BMC Alexandria, 875-876; J. BEAUJEU, La religion romaine à l'apogée de l'Empire I, Paris, 1955, p. 230-232; P.M. FraSER, Ptolemaic Alexandria, I, Oxford, 1972, p. 272275 .

Dionisio usa, per indicare Alessandria, una perifrasi di sei versi (254-259), la più lunga che s'incontra nella Periegesi. Non è il solo caso di omissione volontaria di dati geografici relativi alla propria patria, se è vero che il poeta decide di tacere la presenza del Delta al v. 18, non per ragioni pedagogiche (semplificazione della problematica relativa ai confini dell'Egitto), bensì per "faire disparaître tout ce qui lui est proche" (così P. CouniLlon, Édition critique de la Périégèse de Denys, Université de Langue et Lettres de Grenoble 3 [1983], p. 143). Come che sia, la divisione

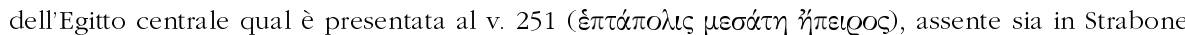
(XVII, 1, 3) sia in Plinio (V, 49), ma non in Tolemeo, lascia pensare che "il s'agit sans doute d'un nouveau découpage administratif, dont Denys et Ptolémée, tous deux Alexandrins, ont dû l'un et l'autre avoir la connaissance directe" (COUNILLON, o.c., p. 178). 
laddove, ad es., più o meno nello stesso torno di secoli Dione Crisostomo considera l'Egitto una semplice appendice $(\pi \varrho \circ \sigma \theta \eta \dot{\gamma} x)$ di Alessandria ${ }^{10}$.

A tali elementi di per sé evidenti si aggiunge il contenuto dell'acrostico dei v. 513-532 $(\Theta \mathrm{EO} \Sigma \mathrm{EPMH} \Sigma \text { EПI A } \triangle \mathrm{PIANOY})^{11}$, che, qualunque valore voglia

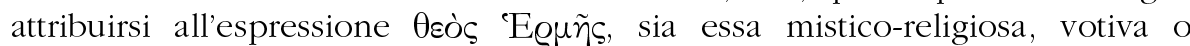
semplicemente un travestimento sincretistico per indicare l'ispirazione del poema, riporta la Descriptio in ambiente egiziano ${ }^{12}$.

Vi è, però, un luogo deputato per sua natura più d'ogni altra sezione di un

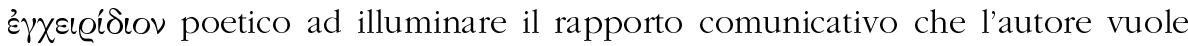
stabilire con il pubblico che avrebbe utilizzato la sua opera e che andrebbe analizzato non tanto con l'analisi delle tecnica impiegata in rapporto con le costanti strutturali riscontrabili nei poemi epici o didascalici, bensì per le sue valenze ideologiche. Nei proemi, in assenza di dedicatari espliciti, va ancora più attentamente indagata quella che Genette definisce l'istanza prefativa, la quale, nel caso di trattati o manuali in versi, diventa imprescindibile, rischio la piatta uniformità degli incipit, tesi, apparentemente, solo ad informare il lettore dell'argomento dell'opera, a danno delle cosiddette "Autorfunktionen" ${ }^{13}$.

10 Cf. Dione Crisostomo, XXXII, 36. In Dionisio, però, io credo, l'elogio dell'Egitto, lungi dall'essere un repertorio di luoghi comuni (così ritiene Counillon, o.c. [n. 9], p. 175-176), rivela l'affetto del patriota.

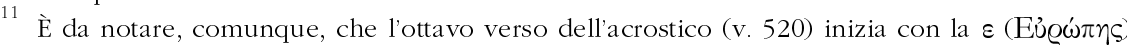

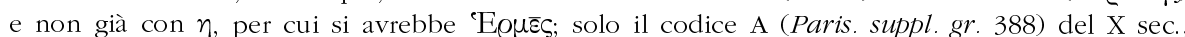
capostipite della cosiddetta "recensione romana", ed il suo unico discendente $\mathrm{V}^{9}$ (Vat. gr. 910, ca.

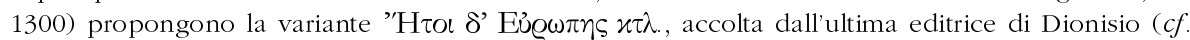
Tsavari, $\Delta$ ıovuoíou [n. 12], p. 72), ma su cui si può discutere: vedi AMATO, l.c. (n. 5), p. 2.

${ }^{12}$ Sui molteplici significati simbolici di Ermes e il suo ruolo nella società greco-romana di età imperiale e tardo-antica, vedi G. FowdEn, Hermès l'Égyptien. Une approche historique de l'esprit du paganisme tardif, trad. fr. Paris, 2000 [Princeton-N.J., 1993²], p. 45-58. Quanto, propriamente, al senso dell'acrostico dionisiano, occorre menzionare le interpretazioni del Leue, l.c. (n. 4) e del Crusius (Jahrb.f. Philol. 137 [1888], p. 525-526), in buona sostanza molto vicine, che ritengono le parole di Dionisio una sorta di formula votiva di datazione relativa al tempo di Adriano, per cui la composizione dell'opera cadrebbe tra il 117 e il 138 d.C. Più precisi, al confronto, risultano gli interventi di A. Diller (The Textual Tradition of Strabo's Geography, Amsterdam, 1975, p. 8) e di J. ALsina ("Panorama de la épica griega tardía", EClás 16 [1972], p. 149) che collocano la Descriptio rispettivamente intorno al 120 e al 124 d.C., anni in cui l'imperatore si iniziò ai misteri di Ermes a Imbro (la seconda datazione, proposta anche da U. BERNAYs, o.c. (n. 8), p. 14, è seguita da H. GärTnER, s.v. "Dionysios" (nr. 30), Kleine Pauly II, col. 73 e da J. SOUBIRAN, Aviénus. Les Phénomènes d'Aratos, Paris, C.U.F., 1981, p. 30, n. 1). Al contrario, per P. Counillon, "Un autre acrostiche dans la Périégèse de Denys", REG 94 (1981), p. 514-522: 517, seguito da

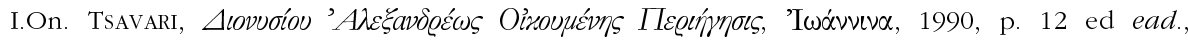
Histoire (n. 4), p. 30-31, dietro l'invocazione ad Ermes si nasconderebbe il favorito di Adriano, Antinoo, la cui morte, avvenuta ad Ermopoli, nei pressi del Nilo, intorno al 130, spinse l'imperatore a recarsi in Egitto e promuovere colà il culto misterico di Ermes-Antinoo.

13 Cf. C. SAntini, N. Scivoletto, Prefazioni, Prologhi, Proemi di opere tecnico-scientifiche latine I, Roma, 1990, p. VI-VII. Sulla funzione dei proemi nella poesia greca, vedi di recente M. FANTUZZI, "Occasioni di performance e generi letterari", in M. FANTUZZI, R. HunTER, Muse e modelli. La poesia ellenistica da Alessandro Magno ad Augusto, Roma / Bari, 2002, p. 3-60; G. WheELER, "Sing, Muse...: The Introit from Homer to Apollonius", CQ 52 (2002), p. 33-49. Cf., 


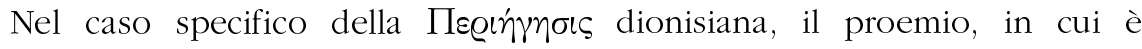
evidente il sapiente gioco intellettualistico e lo sperimentalismo di matrice ellenistica, che dà vita ad un'efficace ed originale "Kreuzung der Gattungen", ben indagata anche di recente ${ }^{14}$, vi sono sincretisticamente annunziante alcune istanze tipiche del background culturale, cui Dionisio appartiene, e che aiutano, pertanto, a collocare meglio la sua poetica nel contesto alessandrino del I-II sec. d.C.

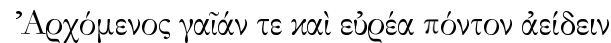

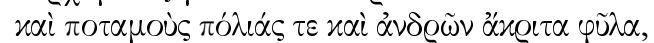

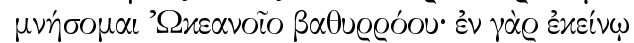

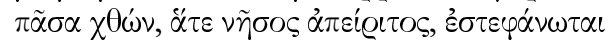

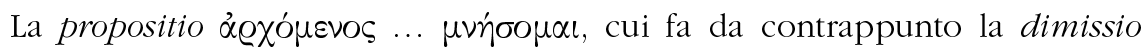

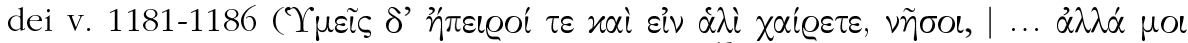

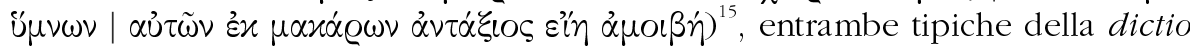
innodica, hanno fatto giustamente concludere che "il poemetto geografico dell'adrianeo Dionigi si presenta sì come epico-didattico ma incorniciato come un inno" ${ }^{, 16}$. Per questo motivo, Dionisio, operando una riscrittura dei modelli anteriori, in particolare di Arato $^{17}$ e di Apollonio Rodio ${ }^{18}$, ma anche di Esiodo ${ }^{19}$,

inoltre, W.H. RACE, "How Greek poems begin", in F.M. Dunn, Th. COLE (ed.), Beginnings in Classical Literature, Cambridge / London, 1992, p. 13-38. Restano, comunque, imprescindibili le riflessioni di G.B. Conte, "Proemi al mezzo", in Miscellanea di studi in onore di Marino Barchiesi I, Roma, 1978, p. 263-273 (= "Proems in the middle", YClS 29 [1992], p. 147-159).

${ }^{14}$ Vedi in particolare O. Vox, "Dionigi Alessandrino e Apollonio Rodio: cornici innodiche", Lexis 20 (2002), p. 153-170. Cf., inoltre, J. STENZEL, De ratione quae inter carminum epicorum prooemia et hymnicam graecorum poesin intercedere videatur, Breslau, 1908, 14; B. EFFE, Dichtung und Lehre. Untersuchungen zur Typologie des antiken Lehrgedichts, München, 1977, p. 192-193; GREAVES, O.c. (n. 8), p. 106-109.

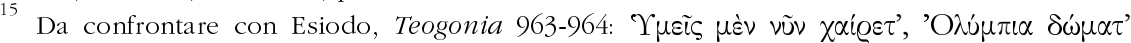

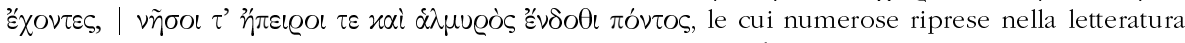
ellenistica sono discusse da O. Vox, l.c. (n. 14), p. 159-160. Personalmente, non vedo così stringente il richiamo ad Arato, 16-18, proposto da Counillon, o.c. (n. 9), p. 318, né tanto meno quello ad Apollonio Rodio, IV, 1773-1781, individuato da Vox, l.c. (n. 14), p. 161-166, ferma restando in Apollonio la probabile dimissio innodica e la motivazione della stessa, analogamente come avviene in Dionisio, con la fine del percorso del viaggio del narratore stesso (qui epico, lì didattico).

${ }_{17}^{16}$ Vox, l.c. (n. 14), p. 153.

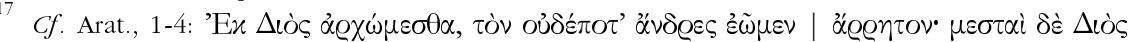

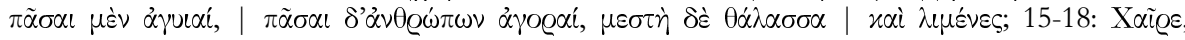

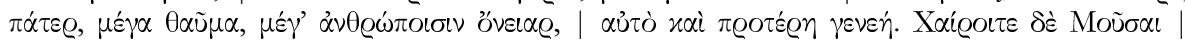

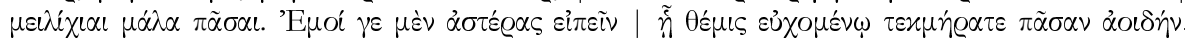
Sulle letture aratee di Dionisio, vedi ora Y. KHAN, "Denys lecteur des Phénomènes d'Aratos", REA 106 (2004), p. 233-246. Cf., inoltre, R. HunTER, "The Periegesis of Dionysius and the Traditions of Hellenistic Poetry", ibid., p. 217-231: 226.

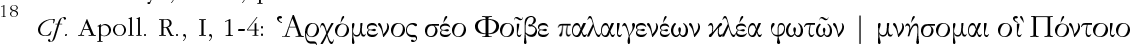

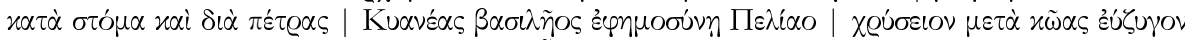

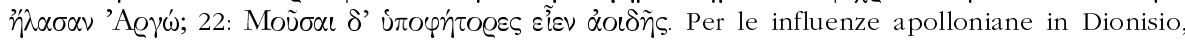
vedi E. AMATO, "Note esegetiche e testuali alla Descriptio orbis di Dionisio d'Alessandria (I)", Arctos 36 (2002), p. 7-17: 9-10; id., "Note esegetiche e testuali alla Descriptio orbis di Dionisio 
caratterizzerebbe la sua Periegesi al tempo stesso come poema didascalico ed epico $^{20}$.

Se da Arato deriva la sostituzione del panteistico Zeus con l'Oceano

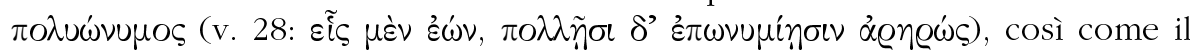
ritardo nell'invocazione alle Muse, che in Arato troviamo ai v. 16-18, in

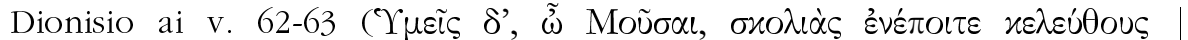

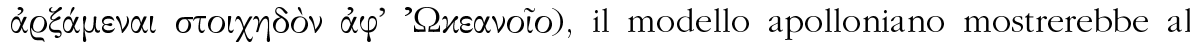
poeta un'altra peculiarità: "anche Apollonio ometteva di invocare in apertura, e si limitava a citarle solo alla fine del proemio, giusto prima di passare alla narrazione, l'impegnativo catalogo degli Argonauti, mai in un semplice auspicio, non apostrofandole dunque con il vocativo". In più, "la dizione

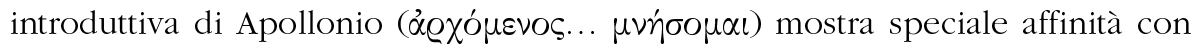

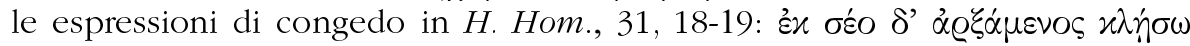

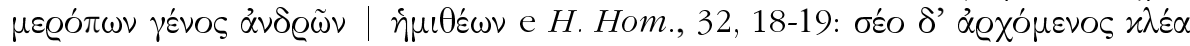

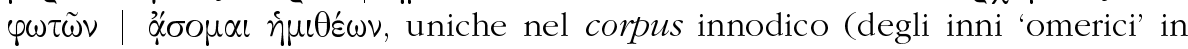
funzione proemiale) ad associare il saluto al dio con l'annuncio di poesia epica eroica" ${ }^{21}$.

Tali interpretazioni, tutte ugualmente legittime e fondate, perdono, però, di vista (o almeno in parte) le novità assolute dell'incipit dionisiano, che nasconde istanze comunicative col lettore ben più originali. Vale a dire che la contaminazione del "modello-genere" didattico (Esiodo) ${ }^{22}$ con il "modelloautore" (Arato e Apollonio Rodio) nel proemio di Dionisio resterebbe inattiva, se non si tentasse di comprenderne i compimenti sostanziali alla luce delle novità strutturali emerse.

Le innegabili somiglianze formulari portano con sé anche diversità ideologiche che non possono essere ridotte unicamente all'esigenza di $\pi$ owi $\lambda i \alpha$, che ha come corollario lo sforzo "um jeden Preis modern zu erscheinen und überraschende Effekte zu erzielen ${ }^{23}$.

d'Alessandria (II), ibid. 37 (2003), p. 10-26: 24-26; C. Cusset, "Denys lecteur d'Apollonios de Rhodes ? L'exemple de la description des fleuves", REA 106 (2004), p. 203-215; HunTER, l.c. (n. 17), p. 218-223.

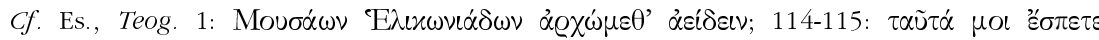

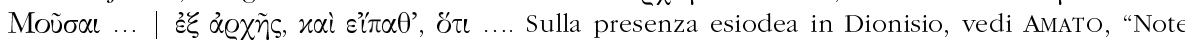
esegetiche I" (n. 18), p. 10-11; id., "Note esegetiche II" (n. 18), p. 25.

${ }^{20}$ Cf. Counillon, o.c. (n. 9), p. 137.

${ }^{21}$ Vox, l.c. (n. 14), p. 156. Sui proemi di Apollonio e Dionisio, vedi anche HunTter, l.c. (n. 17), p. 218. Va, in ogni caso, rilevato, come ottimamente ha messo in luce E. LIVREA, "Il proemio degli Erga considerato attraverso i v. 9-10", Helikon 6 (1966), p. 442-475: 445-446 (ma vedi già U. vON WiLAMOWITZ-MÖLLENDORFF, Hesiods Erga, Berlin, 1928, p. 39-40), che l'affrancamento dalle tradizionali invocazioni innodiche, più oculato e meno vistoso, comincia ad essere operante già in Esiodo, il quale, soprattutto negli Opera, propone di suo alle Muse la materia del canto e non viceversa.

${ }^{22}$ Non sfuggano a tal riguardo le opportune avvertenze di L.E. ROSsI, "La letteratura alessandrina e il rinnovamento dei generi letterari della tradizione", in R. PrETAGOSTini (ed.), La letteratura ellenistica. Problemi e prospettive di ricerca, Roma, 2000, p. 149-161: 155.

${ }^{23}$ Così W. Kroll, Studien zum Verständnis der römische Literatur, Stuttgart, 1924, p. 202203. 
Per intenderci, la portata della rottura dell'incipit arateo, avvertita fin dai contemporanei, tanto da farne circolare varie redazion ${ }^{24}$, non si comprenderebbe se non se ne interpretasse il valore allegorico-filosofico della figura di Zeus; ciò che permise a Cratete di Mallo di difenderne la formula definitiva,

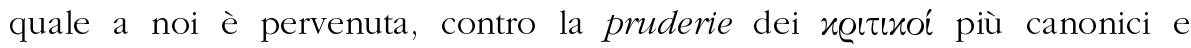
regolari, che intravedevano uno Zeus fabulosus, in aperto contrasto col la consolidata tradizione del proemio musaico ${ }^{25}$. Analogamente, nel caso di Apollonio, l'invocazione ad Apollo ha incidenze sulla destinazione stessa del poema: Apollo, nel sincretismo religioso greco-egiziano, era paragonato, fin dai tempi di Erodoto (II, 156), al dio Horus, venerato in tutto l'Egitto con un grandissimo numero di santuari e con il quale volentieri i faraoni d'Egitto amavano identificarsi ${ }^{26}$. Ne consegue che l'autore delle Argonautiche con tale immagine ha voluto non solo omaggiare la persona del sovrano di turno, ma anche una delle divinità più rappresentative della religione egizia ${ }^{27}$.

Il primo commentatore della formula cletica della Periegesi, il vescovo Eustazio di Tessalonica ${ }^{28}$, differentemente dall'anonimo Scoliaste, cui non era sfuggita l'allusione apolloniana ${ }^{29}$, propone un accostamento, ingiustamente

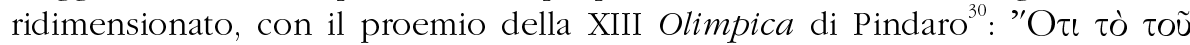

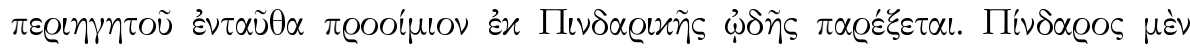

24 Vedi J. Martin, Arati Phaenomena, Firenze, 1956, p. 4.

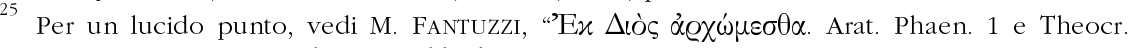
XVII 1", MD 5 (1980), p. 163-172: 166-167. L'ipotesi del MAASS (Aratea. Philologische Untersuchungen, Berlin, 1892, p. 319-320) secondo cui Arato avrebbe recitato il suo poema durante un cenacolo poetico a Cos, sembra essere destinata a rimanere tale: cf. MARTIN, o.c. (n. 24), p. 4; M. ERrEN, Die Phainomena des Aratos von Soloi, Wiesbaden, 1967, p. 15; D. KIDD, Aratus. Phaenomena, Cambridge, 1997, p. 163.

26 Sulla problematica esiste una nutrita bibliografia. Mi limito a ricordare J.L. TONDRIAU, "Rois Lagides comparés ou identifiés à des divinités", CE 23 (1948), p. 127-146; FraSER, o.c. (n. 8), p. 189-193; H. MELAERTS (ed.), Le culte du souverain dans l'Égypte ptolémaique au IÍ siècle avant notre ère, Leuven, 1998. Quanto al noto fenomeno del sincretismo religioso greco-eigizio, vedi D. DiETRICH, "Die Ausbreitung der alexandrinischen Mysteriengötter Isis, Osiris, Serapis und Horus in griechisch-römischen Zeit", Altertum 16 (1968), p. 201-211; R. IsLEY HICKs, "Egyptian elements in Greek mythology", TAPhA 93 (1962), p. 90-108.

${ }^{27}$ Cf. Vox, l.c. (n. 2), p. 165

28 Edizione e traduzione latina in C. MüLLER, Geographi Graeci Minores, II, Parisiis, 1861,

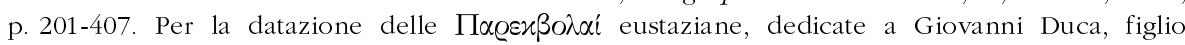
dell'imperatore Andronico Paleologo, ed anteriori a quelle omeriche, vedi A. KAZHDAN, Studies on Bizantine Literature of the Eleventh and Twelfth Centuries, Cambridge / Paris, 1984, p. 115-195. Non è un caso che Eustazio abbia dedicato i suoi Commentari unicamente ad Omero e Dionisio: ciò significa che quest'ultimo era sentito certamente come un emulo del Пoın $\hat{n}_{\text {c }}$ e, soprattutto, che la sua opera rivestisse, per così dire, un'importanza 'ecumenica' (cf. JACOB, l.c. [n. 1], p. 226). Anzi, dal momento che il commento dionisiano venne redatto qualche tempo prima di quello dedicato ad Omero, se ne deduce che agli occhi del vescovo di Tessalonica la lettura e l'interpretazione della Descriptio orbis rappresentasse un'ottima palestra propedeutica all'agone maggiore.

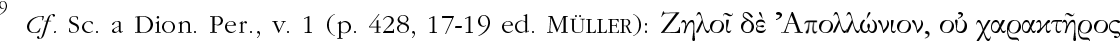

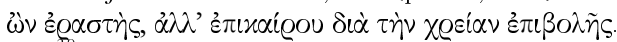

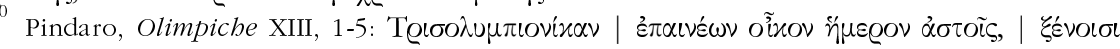

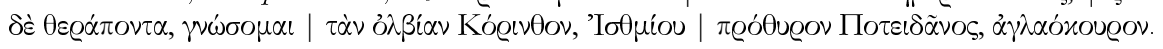




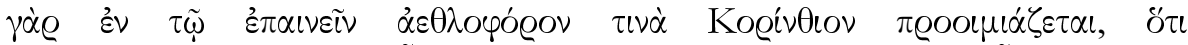

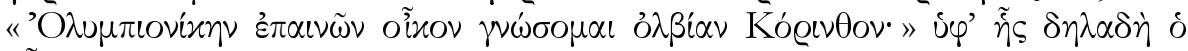

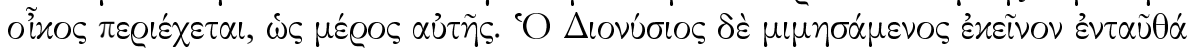

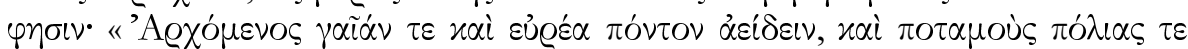

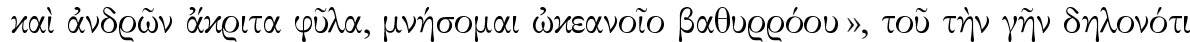

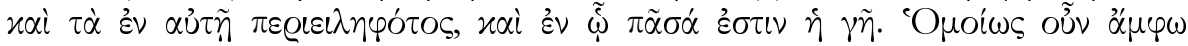

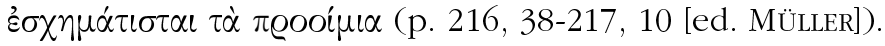

Il richiamo, lungi dal proporre un magro ed infertile parallelo strutturale e

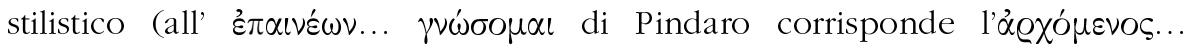
$\mu \nu \eta \dot{\sigma o o} \mu \alpha$ di Dionisio), lascia intuire che al dotto esegeta bizantino non era, forse, sfuggita la caratteristica saliente del proemio della Descriptio. La lode della casata dell'olimpionico Senofonte e del rapporto ospitale che la lega al poeta tebano serve induttivamente a 'riconoscere' nel suo valore la città di Corinto $^{31}$. In Dionisio, dov'è non vi è invocazione alcuna alle divinità dell'Olimpo ellenico, cui in genere non è elusa la conoscenza delle 'gesta' umane $^{32}$, le terre emerse, $\mathrm{i}$ fiumi, il mare e le innumerevoli popolazioni servono, parimenti con un processo induttivo, a 'ricordare' e, dunque, a lodare dinanzi ai lettori l'onnipresenza dell'Oceano 'che scorre profondo', il quale, come lo Zeus stoico dei Phaenomena aratei, pur essendo uno, prende

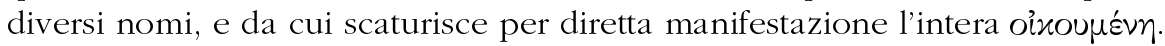

E questo un procedimento tipico dell'innografia e, soprattutto, della tecnica eulogistica, la quale prevede l'esaltazione del laudandus attraverso la presentazione dei suoi poteri e della sua sfera d'influenza (le virtutes) in un mutuo rapporto di scambio ${ }^{33}$, per cui se, ad es., la vittoria di Senofonte dà fama e gloria alla gioventù di Corinto, questa, a sua volta, encomiata per il suo assetto etico-istituzionale e per la sua cura nelle attività agonistiche, risulta come il luogo più adatto per il vittorioso olimpionico che intende rivolgere la

31 Per il senso di ${ }^{2} \omega \dot{\omega} \sigma o \mu \alpha$, vedi A. PUECH, Pindare I. Olympiques, Paris, CUF, 1958, p. 148, n. 1: "Le verbe $\gamma \nu \omega ́ \sigma o \mu \alpha \iota$ ne peut avoir ni le sens de visiter ni le sens causatif, faire connaître, célébrer, qu'on lui donne ordinairement ici. Pindare reconnaît en cette famille les traits caractéristiques du génie Corinthien". Cf., inoltre, L. LeHnus, Pindaro. Olimpiche, Milano, 1981, p. 204 .

Ricordo, tra i contemporanei ed i successori di Dionisio, l'invocazione a Calliopea da parte di Trifiodoro, delle Ninfe di Troia da parte di Colluto, di una divinità non specificata da Nonno. Al contrario, nei Cynegetica di Oppiano di Apamea, così come negli Halieutica dell'omonimo di Anazarbo vi è la menzione dei rispettivi dedicatari (Caracalla ed Marco Aurelio). Mancano, invece, di una vera e propria praefatio i Posthomerica di Quinto Smirneo. In precedenza, Nicandro aveva dedicato i Theriaca ad Ermesianatte, gli Alexipharmaca a Protagora.

33 Cf. C.O. PAVESE, "L'inno rapsodico", in A.V., L'inno tra rituale e letteratura nel mondo antico. Atti di un Colloquio. Napoli 21-24 ottobre 1991, Roma, 1993, p. 155-178: 166-167. Sulla struttura dell'inno poetico, vedi inoltre R. WÜNSCH, s.v. "Hymnos", RE IX (1914), col. 140-183: 145; P. COLlaRT, "L'invocation d'Isis, d'après un papyrus d'Oxyrhynchos", REgypt 1 (1919), p. 93-100: 96; J.M. BREMER, "Griekse hymnen", Lampas 12 (1979), p. 95-110: 96 (= "Greek Hymns", in H.S. VerSnel (ed.), Faith, Hope and Worship, Leiden, 1981, p. 193-214); P. WüLfIng, "Hymnos und Gebet. Zur Formengeschichte der älteren griechischen Hymnendichtung", StudClas 20 (1981), p. 21-31: 23; W.D. Furley, J. MAARTEN BREMER, Greek Hymns I. The Texts in Translation, Tübingen, 2001, p. 50-64. In particolare per Pindaro, vedi P. ANGELI BERNARDini, Mito e attualità nelle odi di Pindaro. La Nemea 4, l'Olimpica 9, l'Olimpica 7, Roma, 1983, p. 19-37. 
propria preghiera a Zeus. Né può dimenticarsi nella dinamica della poesia laudativa di età arcaica il ruolo rilevante del pubblico costituito da phroneontes che comprendono e penetrano a fondo il messaggio del laudator. Come'è stato messo ottimamente in luce, "premesso che nell'ideologia della poesia celebrativa lo elogio non può essere tributato da uno qualunque, ma solo da un sophos..., appartenente a una categoria di individui che emergono tra gli altri per le loro qualità, anche gli ascoltatori, cioè il pubblico al quale il poeta si rivolge, debbono saper capire la lode ${ }^{, 34}$. In tal senso, dunque, alla luce delle innegabili e profonde differenze, va indagato se le scelte di Dionisio, come quelle di Pindaro, vengano fatte sulla base di un criterio di recettività da parte di chi ascolta.

Dicevamo che Dionisio, a differenza di Arato e di Apollonio Rodio, incentra il proprio progetto didascalico esclusivamente sull'azione del 'ricordare' ( $\mu \nu \eta \dot{\sigma o} \mu \alpha \iota)$, motivo centrale del genere epico, "perché senza canto epico le grandi gesta del passato sono destinate all'oblio",35, facendo di se

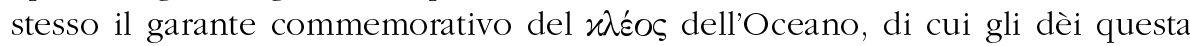
volta non sono a conoscenza.

Se il proemio di Dionisio non ha conosciuto certo quella fluttuante dinamica redazionale che abbiamo incontrato per i $\Phi \alpha \iota v o ́ \mu \varepsilon v \alpha$, è pur vero che nel momento in cui Avieno e, quindi, Prisciano decisero di proporre al pubblico di lingua latina la Descriptio orbis si trovarono costretti a diluire l'intenzione polemica o, comunque, innovativa dell'incipit dionisiano con la canonica dedica di un poeta cortigiano e l'invocazione alle divinità ${ }^{36}$.

Perché tale cambiamento? Secondo il Greaves: "Dionysius has combined the expectations of poetic form with his geographical theme in such a way that what begins as though it might be a commemoration of deity is completed as geographical outline... Dionysius, however, makes no implications regarding the Ocean as deity, but treats it exclusively as a geographical feature ${ }^{37}$. È per questo, dunque, che i versificatori latini, presi, probabilmente, dall'imbarazzo dinanzi all'assenza di un tradizionale $\pi$ gooí $\mu$ ı v musaico, senza neppure l'indicazione del destinatario, hanno pensato di invocare posticciamente ad inizio del proprio poema divinità tipiche del pantheon classico.

34 Così ANGELI BERNARDini, o.c. (n. 33), p. 32.

35 Così R. Hunter, "Le 'Argonautiche' di Apollonio Rodio e la tradizione epica", in FANTUZZI, HUNTER, o.c. (n. 13), p. 121-175: 125.

36 Se Avieno aggiunge l'invocazione alle Muse e ad Apollo (v. 1-10: Qua protenta iacent vastae divortia terrae let qua praecipiti volvuntur prona meatu / flumina per terras, qua priscis inclyta muris / oppida nituntur, genus boc procul omne animantum / qua colit, Aoniis perget stilus impiger orsis. / ardua res, musae. deus, en deus intrat Apollo / pectora, fatidicae quatiens penetralia Cirrhae. / Pierides, toto celeres Helicone venite, / concinat et Phoebo vester chorus; Oceanumque, / carminis auspicium, primum memorate camenae.), Prisciano si rivolge al naturae Genitor (v. 1-4: Naturae genitor, quae mundum continet omnem, / annue, rex coeli, positum telluris et undae, / in quas imperium mortalibus ipse dedisti, / materiae tantae me premere carmine digno)

37 GREAVES, o.c. (n. 9), p. 108. 
Al contrario, possiamo affermare che l'istanza religiosa nel proemio dionisiano è quanto mai operante. Non sto certo a soffermarmi sul ruolo giocato dall'Oceano nella cosmogonia e nella mitologia greca, così come nell'orfismo e nei sistemi teogonici di molte altre civiltà antiche ${ }^{38}$, benché la traccia orfica, considerando l'influenza su Dionisio delle Zeus dei Phaenomena, non sarebbe totalmente da sottacere ${ }^{39}$. Ma, un punto che va sviluppato è quello che potrebbe equiparare, nella prospettiva di una destinazione anche alessandrina del poema, l'Oceano all'egiziano Nun, l'oceano primordiale della creazione da cui tutto deriva ed in cui tutto torna, in una visione panteistica dell'universo cui è intimamente legata la religione egizia.

Nell'ambito delle numerose cosmogonie che contraddistinguevano la storia religiosa dell'antico Egitto (ve ne è quasi una per ogni singolo nomo), tutte hanno in comune l'idea di un oceano primordiale indistinto, un'acqua abissale, assoluta, padre di tutti gli dèi e del creato, dalla quale emerge poi una collina, primo appoggio per il demiurgo che dà così inizio alla creazione.

Se, tuttavia, indaghiamo a fondo, si scopre che, tra i centri più importanti della speculazione cosmogonica egizia, quello di Ermopoli offre, rispetto all'elipolitano ed al menfitano, un sistema in cui il dio creatore, Ermes-Thot, con la sua parola crea un'ogdoade di divinità allegoriche. Queste depongono su un'isola primordiale (Tatenen), in mezzo al Nun, visto, dunque, come un principio attivo di carattere divino, un uovo dal quale spunta il Sole (AtumRa). Si tratta di un concezione materialistica della genesi in parte differente dalla cosmologia elipolitana, dove la forza attiva della creazione è rappresentata da Atum-Ra (il Sole), venuto al mondo da solo e la cui attività

\footnotetext{
38 Per i numerosi passi, vedi G. RICCIARDELu, Inni orfici, Milano / Verona, 2000, p. 526. Mi limito unicamente a ricordare che per Omero dall'Oceano, che circonda la terra, deriva il mare e tutta l'acqua della terra (Iliade XXI, 196-197), è origine degli dèi (Il. XIV, 201; 302) e non partecipa alle riunioni delle altre divinità (Il. XX, 7). Al contrario, in Esiodo Oceano è figlio di Cielo e Terra (Teog., 133) e da esso avrebbero origine solo i fiumi (Teog., 337-338). In altre teogonie, il ruolo dell'Oceano doveva essere ancora più importante: $c f$. Orph., fr. 21 (ed. BERnABÉ). Tuttavia, stando all'interpretazione che Damascio fornisce nel de principiis $(124=\mathrm{III}$,

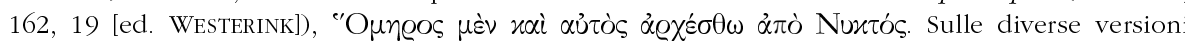
dell'origine del mondo in Grecia antica, vedi K. KERÉNYI, Gli dei e gli eroi della Grecia, trad. it. Milano, 1989 [1963리, p. 29-32; R. GRAVES, I miti greci, trad. it. Milano, 1979³ , p. 27-28. Cf., inoltre, H. HerTER, s.v. "Okeanos" (nr. 1), RE XVII/2 (1937), col. 2308-2361; A.F. LOSSEW, "De chao quid antiqui senserint", Meander 12 (1957), p. 283-293; U. BIANCHI, Teogonie e cosmogonie, Roma, 1960; M. MARCONI, "Can the cosmogony of the Greeks be reconstructed?", HR 1 (1961), p. 274280; J. RUDHARDT, Le thème de l'eau primordiale dans la mythologie grecque, Berne, 1971; J. RAMIN, Mythologie et géographie, Paris, 1979, p. 17-26; S. VILATTE, L'insularité dans la pensée grecque, Paris, 1991. Per le immagini dell'ecumene come isola in altre culture antiche, vedi A. SEPPILl, Sacralità dell'acqua e sacrilegio dei ponti, Palermo, 1990², p. 92-126.

39 Vedi in particolare [Orph.], H. 13: "Invoco Oceano, padre immortale, che sempre è / origine degli dèi immortali e degli uomini mortali, / che ondeggia intorno al cerchio che delimita la terra; / dal quale derivano tutti i fiumi e tutto il mare / e i santi umori ctoni della terra che scorrono dalle sorgenti. / Ascolta, beato, molto felice, grandissimo mezzo di purificazione degli dèi, / caro termine della terra, principio del cielo, dalle umide vie, / vieni benevolo agli iniziati sempre lieto" (trad. G. RICCIARDELLI).
} 
sessuale è alla base della differenziazione dell'informe Nun; di sicuro profondamente diversa da quella biblica, poiché per la cosmogonia di Ermopoli tutto, anche il demiurgo, il creatore, scaturisce dal Nun preesistente ${ }^{40}$.

Ecco, allora, che nel suo proemio Dionisio, dove vi è menzione dell'Oceano "che scorre profondo" nel cui abbraccio l'ecumene appare appunto come un'isola sconfinata ( $\nu \tilde{n} \sigma 0 \varsigma \hat{\alpha} \pi \varepsilon i \varrho \alpha \tau O c)$, ha voluto sì omaggiare la tradizione omerica ${ }^{41}$ riconosciuta dalla geografia greca ${ }^{42}$, ma, nell'indirizzarsi ad un preciso ambiente culturale-religioso ha inteso altresì elogiare ed

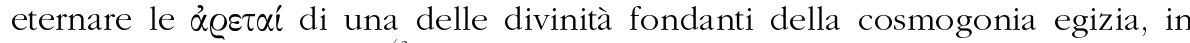
particolare ermopolitana ${ }^{43}$. Non è un caso, credo, che il poeta dedichi all'Oceano ed alle sue virtutes (il potere e la sfera d'influenza) ${ }^{44}$ ben sessantuno versi (il che corrisponde a circa il 20\% dell'intero poema), prima della tradizionale invocazione alle Muse da cui viene la sacralità del canto.

Se è pur vero che la Periegesi è come incorniciata in moduli formulari tipici dell'innografia, andrà senz'altro notato che nel caso dell'incipit si tratta di un modulo 'speciale', visto che 1) il nome del dio cantato in prima persona

${ }^{40}$ Sull'argomento, vedi S. SunERON, J. YOYOTTE, "La naissance du monde selon l'Égypte ancienne", in A.V., La naissance du monde, Paris, 1959, p. 17-91; E. HoRnung, Der Eine und die Vielen. Ägyptische Gottesvorstellungen, Darmstadt, 1971.

1 Cf. Om., Il. XVIII, 399; 607-608; Odissea XX, 63-65. L'autorità di Omero, richiamata da Strabone in I, 1, 3 e 7, è riconosciuta dalla maggior parte dei geografi antichi: vedi H.F. TOZER, $A$ History of ancient Geography, Cambridge, 1897, p. 250; A.M. BIRASCHI, "Strabone e la difesa di Omero nei Prolegomena", in F. PrOnTERA (ed), Strabone. Contributi allo studio della personalità e dell'autore I, Perugia, 1984, p. 129-153.

${ }^{42} C f$. Strab., II, 5, 5, in cui si legge che l'ecumene, bagnata dal mare, è simile ad un'isola

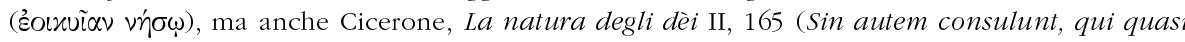
magnam quandam insulam incolunt, quam nos orbem terrae vocamus, etiam illis consulunt, qui partes eius insulae tenent, Europam, Asiam, Africam.); Repubblica VI, 20 (Omnis enim terra, quae colitur a vobis, angustata verticibus, lateribus latior, parva quaedam insula est circumfusa illo mari, quod 'Atlanticum', quod 'magnum', quem 'Oceanum' appellatis in terris). La concezione risalente ad Omero (vedi supra, n. 38) che l'oixounévn sia identificabile con un'immensa isola ricorre anche nella visione scientifica di Eratostene e di Posidonio: cf. A. Thalamas, La géographie d'Ératosthène, Versailles, 1921, p. 219-223; G. AUJAC, "Les représentations de l'espace géographique ou cosmologique, dans l'Antiquité", Pallas 28 (1981), p. 4-5. Vedi, inoltre, per l'antica concezione della terra rotonda, circondata dal mare, Eschilo, Prometeo 138-139; Euripide, Oreste 1377-1379; [Orph.], H., XI, 15; LXXXIII, 3; fr. 287 (ed. Bernabé); Pomponio Mela, I, 4; Plinio il Vecchio, Storia Naturale II, 242. Ma, in Strabone, così come in Cicerone, non vi è alcuna visione panteistica dell'Oceano. A criticare l'opinione dell'insularità del mondo abitato furono Erodoto (II, 21-23; IV, 36, 2) ed Ipparco (ap. Strab., I, 1, 9), ma anche Aristotele (meteor., 362b) e Gemino (astr. XVI, 4). Sull'intera problematica, vedi A. LESKY, Thalatta. Der Weg der Griechen zum Meer, Wien, 1947, p. 87; F. PRONTERA, "Prima di Strabone: materiali per uno studio della geografia antica come genere letterario", in id., o.c. (n. 41), p. 239-249; A. BALlabriga, Le Soleil et le Tartare. L'image mythique du monde en Grèce archaïque, Paris, 1986, p. 66-67; J. Romm, The Edges of the Earth in Ancient Thought. Geography, Exploration, and Fiction, Princeton, 1992, p. 32-44; F. BORCA, Terra mari cincta. Insularità e cultura romana, Roma, 2000, p. 35-52.

43 Secondo Arriano (India XVIII, 11) e Diodoro Siculo (XVII, 104, 1), Alessandro Magno fece costruire un tempio in onore di Oceano e sua moglie Teti dinanzi al delta del Nilo.

${ }^{44}$ Non esistendo le yovaí dell'Oceano, è chiaro che la struttura innodica è alquanto evanescente, né sarebbe stato facile crearla. 
(il tipo più frequente negli inni, indicato da Carlo Odo Pavese con: cano)

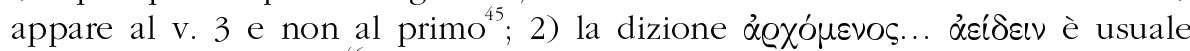

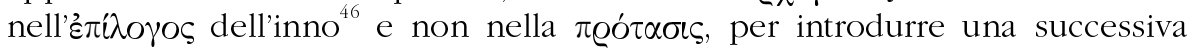
rapsodia eroica, quando, cioè, il poeta, concluso il prooimion, passa alla oime $^{47}$. Ciò può voler dire che Dionisio, nell'ambito di un canto in onore

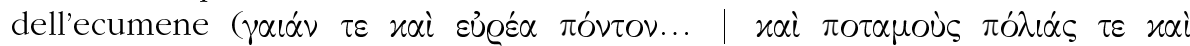
$\dot{\alpha} \nu \delta \varrho \tilde{\omega} \nu \varphi \tilde{u} \lambda \alpha$ ), ha voluto ritagliare uno spazio a sé per l'elogio dell'OceanoNun, usando il modulo formato dall'espressione $\mu \nu \eta \dot{\sigma o} \mu \alpha \iota$ ' $\Omega \varkappa \varepsilon \alpha \nu o \tilde{o}$ $\beta \alpha \theta$ uegóou ${ }^{48}$, così come Pindaro, per ritornare all'accostamento per primo proposto da Eustazio, ha inteso cantare la vittoria del duplice olimpionico Senofonte per poter induttivamente celebrare il valore della città di Corinto.

Sotto tale angolatura, anzi, come semplice ipotesi di lavoro ci si potrebbe spingere ulteriormente avanti e chiedersi se sotto Dionisio non si nasconda realmente un adepto dell'ermetismo imperiale, visto il ruolo rilevante assunto dall'Oceano primordiale nell'ogdoade ermopolitana ${ }^{49}$. Lo stesso acrostico dei

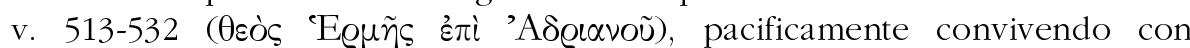
l'interpretazione che vede in esso un riferimento al processo di osirizzazione di Antinoo ${ }^{50}$, potrebbe rappresentare, allora, la professione di fede del nostro poeta, il quale si assume il compito di magnificare il nome di Ermes nell'impero di Roma ${ }^{51}$.

45 Cf. PAvese, l.c. (n. 33), p. 160-162. Tale ritardo, non riscontrabile negli inni, è forse dovuto dalla maggiore estensione dell'annuncio del tema (terra, mare, fiumi, città, popoli) rispetto alla proposizione di un esordio tradizionale, dedicato generalmente ad un'unica divinità.

46 Per i paralleli, vedi supra.

47 Cf. F. CÀssola, Inni omerici, Milano, $1994^{2}$ [1975], p. XVII-XVIII; PAVESE, l.c. (n. 33), p. 164. Nel caso della Descriptio, mancando il congedo alla fine della lode all'Oceano, non è possibile intravedere, com'è per la Teogonia di Esiodo, un esempio della sequenza prooimion-oime.

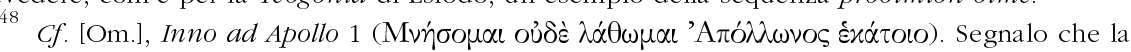

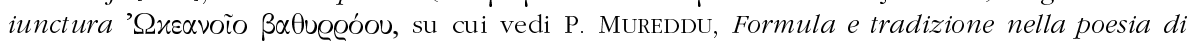
Esiodo, Roma, 1983, p. 30, compare nelle medesime sedi in [Om.], Inno a Mercurio, 185.

49 Per la presenza dell'Oceano in Corpus Hermeticum XIII, 17, in cui vi è menzione della creazione dell'universo e della fuoriuscita delle acque dalla materia liquida primordiale per

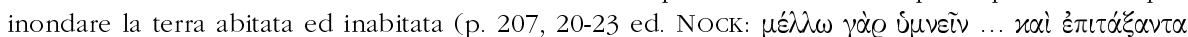

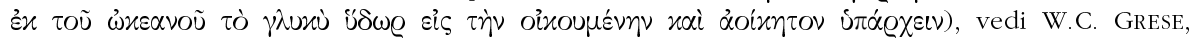
Corpus Hermeticum XIII and Early Christian Literature, Leiden, 1979, p. 175 e D.J. MEREDITH WhiteHOUse, The Hymns of the Corpus Hermeticum: Forms with a diverse functional History, Ph.D., Harvard University, 1985, p. 296-297; 305, che confronta il passo in questione con vari inni egiziani. L'influenza egizia negli inni e nella letteratura ermetica è ben indagata da J.-P. MAHÉ, Hermès en Haute-Égypte. Les textes hermétiques de Nag Hammadi et leurs parallèles grecs et latins I, Québec, 1978, p. 23-26; 33-38.

50 Vedi supra, n. 11. Ricordo che il decreto dell'incamminarsi nell'aldilà per il favorito di Adriano, quale si legge sul terzo lato dell'obelisco del Pincio, testimonia il rispetto di tutti gli abitanti di Ermopoli ed invoca l'intervento del "signore della parole divine" (Thot = Ermes) affinché ringiovanisca l'anima del defunto. Una traduzione italiana annotata si legge ora in E. BRESCIANI, Testi religiosi dell'antico Egitto, Milano, 2001, p. 444-445.

51 L'acrostico potrebbe avere il seguente significato lapidario: "Il dio Ermes esiste nel regno

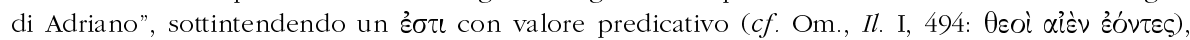

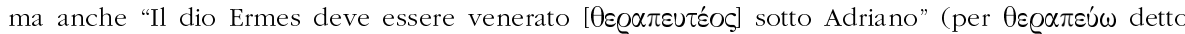
del culto divino vedi, e.g., [Om.], Inno ad Ap., 390). Mi sembra utile indicare in tal senso un 
La centralità di Alessandria nella diffusione dell'ermetismo in età imperiale potrebbe essere garanzia del contatto che Dionisio ebbe con le correnti sincretistiche del dio Ermes, in un periodo in cui l'incontro fra tradizioni elleniche e religione egiziana si trovò ad esprimere la sua carica massima ${ }^{52}$.

Che valore dare, in tal senso, alla testimonianza della Suida secondo cui il padre del nostro Dionisio fu discepolo $\left(\mu \alpha \theta \eta \tau \eta^{\prime}\right)$ del sacerdote egizio e filosofo stoico Cheremone $e^{53}$ ? È risaputa l'importanza dell'attività di tale personaggio $^{54}$, divenuto precettore di Nerone nel 48 d.C., per ciò che riguarda il processo di diffusione degli Hermetica al di fuori dell'Egitto. Del resto, il rapporto delle dottrine religiose e filosofiche professate da Cheremone con l'ermetismo è assicurato, a quanto pare ${ }^{55}$, dal rinvio che Porfirio fa ad una perduta opera dal titolo $\sum \alpha \lambda \mu \varepsilon \sigma \chi \iota v \iota \alpha x \alpha ́$ per un maggiore ragguaglio sul

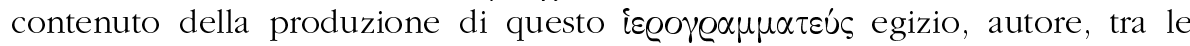

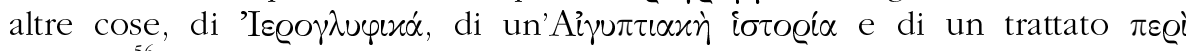

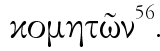

Se tale dato non può affatto confortare l'ipotesi di appartenenza di Dionisio all'ermetismo, non esclude neppure il probabile contatto che il poeta ebbe con questa dottrina tramite il padre. Non si dimentichi, inoltre, che stando alle fonti tardo-antiche il Periegeta sarebbe stato autore anche di uno

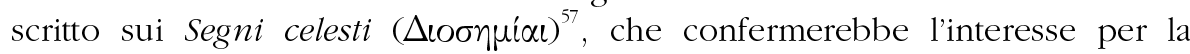

raffronto possibile negli inni acrostici in lingua accadica. In uno, in particolare, dedicato alla città di Babilonia, la frase che viene fuori dalla lettura dell'acrostico è la seguente: "Nelle quattro il suo nome", vale a dire: "Nelle quattro [regioni del mondo io voglio magnificare] il suo nome" ([i]-na ar-ba [kibräti lušanik] zi-kir-su): cf. L. CAGNI, "L'inno in area mesopotamica", in A.V., L'inno (n. 33), p. 13-31: 28. È certo, comunque, che Dionisio abbia inteso dedicare con questo acrostico il suo poema a Dionisio (per l'uso del nominativo, in luogo del dativo o di ઘı̣ + acc., a voler indicare il nome del dedicatario negli acrostici di composizioni encomiastiche, vedi Dioscoride, IV

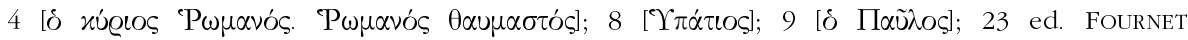
$[\Theta \varepsilon o ́ \delta \omega \varrho O c]$ ).

52 Per un'attenta ricostruzione, vedi FOWDEN, o.c. (n. 12), p. 237-246.

53 Vedi supra, n. 5.

54 Su cui vedi A. BARZANÒ, "Cheremone di Alessandria", ANRW II, 32.3 (1985), p. 1981-2001; M. Frede, "Chaeremon", ibid. 36.3 (1989), p. 2067-2103; R. GOUlET, s.v. "Cheremon", DPhA II (1994), p. 284-286; A.M. URSO, "La letteratura astrologica: gli autori", in I. MASTRAROSA, A. ZuMBO, Letteratura scientifica e tecnica di Grecia e Roma, Roma, 2002, p. 111-137: 114

55 Cf. FOWDEN, o.c. (n. 12), p. 208-209; FrEDE, l.c. (n. 56), p. 2085.

56 Cf. Porfirio, Epist. Aneb. II, 12 = Cheremone, fr. 5 (ed. VAN DER HORST). Vedi anche Giamblico, Myst. VIII, 4 = Cherem., fr. 9 (ed. VAN DER HORST), dove è chiaramente detto che $\tau \dot{\alpha} \tau \Xi$

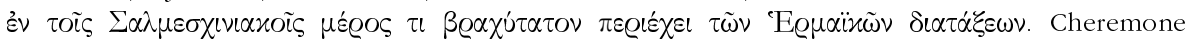
dovette probabilmente occuparsi nelle sue opere di cosmogonia e mitologia egizia, come lascerebbe intendere la testimonianza di Porph. ap. Eusebio, Prep. Evang. III, 11, 45-13, 2 (ed. MRAS $)=$ Cherem., fr. 17D (ed. VAN DER HORST).

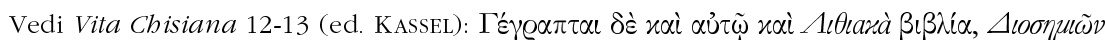

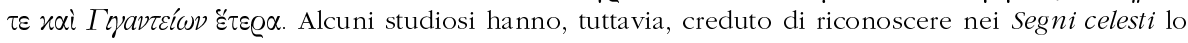
scritto Sulla metereologia, che il lessico Suda attribuisce a Dionisio di Corinto $(\Delta 1177=$ p. 110 [ed. ADLER]): secondo tali criteri l'autore dell'opera sarebbe Dionisio Periegeta ed il lessicografo avrebbe fatto grande confusione fra i vari scrittori di nome Dionisio ( $c f$. G. KNAACK, s.v. "Dionysios", RE IX [1903], col. 923). 
letteratura astronomica o astrologica (non sappiamo) affatto estranea all'ermetismo. Ugualmente, l'interesse per la mineralogia, in particolare per i poteri magici e medicamentosi provenienti dalle pietre, di cui Dionisio poteva offrire una tangibile testimonianza nei suoi $\Lambda_{\imath} \theta \iota \alpha x_{\alpha} \alpha^{58}$, ci riconducono ad un ambiente culturale improntato all'orfismo e all'ermetismo. Quanto alla Descriptio, le modalità di allocuzione nei confronti del discepolo-lettore che incontriamo nel corso dell'opera consentono dei raffronti, attese le profondissime diversità di contenuto e di forma, con analoghe testimonianze d'insegnamento ricavabili dagli Hermetica.

È tipico, ad es., dei dialoghi ermetici il rapporto esclusivo del maestro con un giovane discepolo non nominato, così come la consapevolezza che la relazione che si instaura tra i due non è che un anello di una lunga catena, da passare in successione di discepolo in discepolo ${ }^{59}$. Ancora, vi è espressa la promessa di guidare l'adepto per combattere l'ignoranza ${ }^{60}$, unita al votodovere che il discepolo segua e apprenda gli insegnamenti sacri ${ }^{61}$.

Ebbene, il destinatario della Descriptio di Dionisio non viene mai nominato $^{62}$ ed ha tutta l'aria di essere un discepolo guidato dal suo maestro, unico depositario di una verità da diffondere, a sua volta, tra gli "ignoranti" o, comunque, da passare ad altri in una sorta di catena intellettuale ${ }^{63}$. La promessa di rendere il giovane 'adepto' forte di quei contenuti annunciati è garantita da parte del $\delta เ \delta \alpha ́ \sigma \varkappa \alpha \lambda o \subseteq$ dall'uso del futuro ${ }^{64}$, da parte del $\mu \alpha \theta \eta \tau \dot{n} \varsigma$ dal rispetto del suo compito, che è quello di prestare attenzione ad apprendere $^{65}$. Dionisio, infine, alla stregua dell'anonimo maestro che ne L'Odgoade e l'Enneade chiede al Dio di concedere al discepolo la "verità nell'immagine", la possibilità, cioè, della $\theta \varepsilon \omega Q \alpha^{66}{ }^{66}$ ovvero, come si legge

58 I Letıaxá di Dionisio in tre libri, il cui titolo si ricava con certezza dal testo della Vita

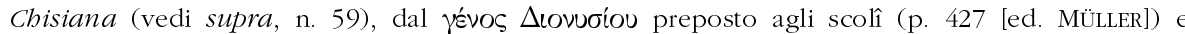
dall'epistola ad Ioannem Ducam di Eustazio (p. 215, 6-14 [ed. MüLLER]), rientravano probabilmente in quel filone della letteratura cosiddetta "paradossografica", al limite tra lo scientifico e il prodigioso, che ricercava nello studio delle pietre preziose il rimedio contro malattie strane o filtri magici, di cui non pochi esempi sono giunti fino a noi. In effetti, all'interno delle diverse correnti che caratterizzano gli scritti $\pi \varepsilon Q i \lambda_{i} \theta \omega \nu$ (scientifico-superstiziosa, magico-sincretica, astrologica, allegorica) nulla impedisce di poter considerare il perduto scritto di Dionisio un esempio del secondo genere, viste anche le sue origini alessandrine, il che ne farebbe un prodotto del sincretismo magico-astrologico orientale, risalente a Zoroastro, ai Magi ed ai Caldei.

59 Sulla problematica, cf. A.J. Festugière, La révélation d'Hermès Trismégiste II, Paris, 1949, p. 31-34; MAHÉ, o.c. (n. 49), p. 31; 89; FOWDEN, o.c. (n. 12), p. 230-233.

Cf. $C H, 1,26$.

61 Cf. Ogdoade e Enneade $\left(N H \mathrm{VI}^{6}\right)$, p. 55, 19-20.

62 Dionisio si rivolge al suo auditor sempre e solo alla seconda persona.

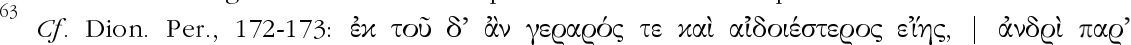

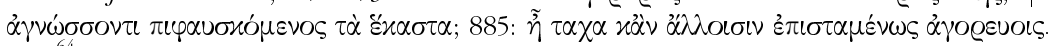

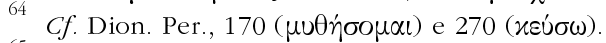

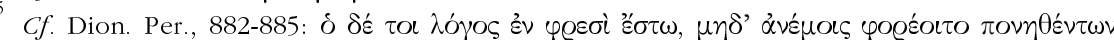

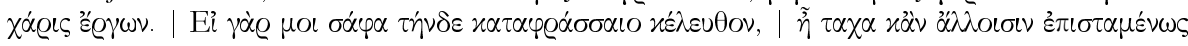

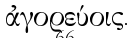

Cf. Ogdoade ed Enneade $\left(N H \mathrm{VI}^{6}\right)$, p. $57,3$. 
altrove, dell'ơ $\psi \iota^{67}$ veritiera dell'oggetto ${ }^{68}$, sembra essere molto attento a presentare l'immagine $(\partial \pi \omega \pi \hat{\eta})$ dell'ecumene come quanto più veritiera, onde evitare le critiche di chi potrebbe tacciare la sua narrazione di falsita ${ }^{69}$.

Concludendo, è chiaro che le osservazioni finora condotte non vanno affatto intese come testimonianza della profonda ispirazione egizia od ermetica del poema di Dionisio. Piuttosto, per rispondere alla domanda inizialmente posta, gli spunti di religiosità egizia che possono rinvenirsi nel corso del proemio dionisiano dovranno essere interpretati come il richiamo ad una comune origine, ad un'atmosfera, che certo non poteva essere esclusa dal background alessandrino dei primi secoli dell'èra comune e che certo Dionisio, in quanto poeta originario di Alessandria, non poteva non tener presente $^{70}$. Soprattutto, esse sono indizio di quella pluralità di approcci ermeneutici che si richiedono a testi, quali la Descriptio orbis, ricchi di implicazioni contenutistiche ed in cui l'intero patrimonio culturale greco si riversa per incontrarsi con le culture del Mediterraneo antico ${ }^{71}$.

Département des Sciences de l'Antiquité

Eugenio AMATO ${ }^{72}$

16 , rue Pierre-Aeby

CH -1700 FRIBOURG

e-mail: Eugenio.Amato@unifr.ch

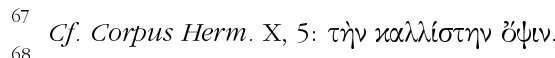

68 Sulla complessa problematica, vedi MAHÉ, o.c. (n. 49), p. 102-103; 109

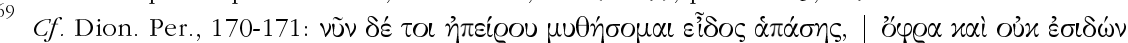

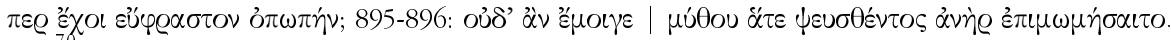

I frustuli di un poema epico dedicato a Mercurio mundi et Hermupolis Magnae conditor si leggono in P.Argent. 481 del IV sec., attribuito dal Wyss (MH 6 [1949], p. 194) al poeta elipolitano Antimaco, di cui vi è notizia nella Suda ( $c f$. E. HeiTsch, Die griechischen Dichterfragmente der römischen Kaiserzeit, I, Göttingen, 1963, p. 82-85).

${ }^{71}$ L'invito a considerare la Descriptio orbis di Dionisio un'opera "ouverte à la politique et au monde contemporain" e non "une oeuvre d'érudition intemporelle" viene di recente lanciato anche da E. BOwIE, "Denys d'Alexandrie : un poète grec dans l'empire romain", REA 106 (2004), p. $117-185$.

72 Durante la correzione delle bozze del presente articolo è apparso il seguente volume: E. Amato, Dionisio di Alessandria. Descrizione della Terre abitata, Milano, 2005. In esso, porto ulteriori argomenti a favore della tesi qui esposta, soffermandomi in particolare sul ruolo svolto da Dionisio ad Alessandria in occasione della visita di Adriano tra il 130 et il 131. A tal proposito buone osservazioni erano già state avanzate da A. Leo in un contributo di cui ho avuto troppo tardi notizia ("La Periegesi di Dionigi d'Alessandria e il viaggio di Adriano in Egitto", Rudiae 13-14 [2002-2002], p. 145-174) 